\title{
2-Cocycles of the Lie superalgebras of Weyl type ${ }^{1}$
}

(appeared in Comm. Algebra, 33 (2005), 2991-3007)

\author{
Guang'ai Song and Yucai Su \\ Department of Mathematics, Shanghai Jiao Tong University, Shanghai, 200240, China \\ E-mail: gasong@sjtu.edu.cn,ycsu@sjtu.edu.cn
}

\begin{abstract}
In a paper by $\mathrm{Su}$ and Zhao, the Lie algebra $\mathcal{A}[\mathcal{D}]=\mathcal{A} \otimes \mathbb{F}[\mathcal{D}]$ of Weyl type was defined and studied, where $\mathcal{A}$ is a commutative associative algebra with an identity element over a field $\mathbb{F}$ of arbitrary characteristic, and $\mathbb{F}[\mathcal{D}]$ is the polynomial algebra of a commutative derivation subalgebra $\mathcal{D}$ of $\mathcal{A}$. The 2-cocycles of a class of $\mathcal{A}[\mathcal{D}]$ were determined by $\mathrm{Su}$. In the present paper, we determine the 2-cocycles of a class of Lie superalgebras of Weyl type over a field $\mathbb{F}$ of characteristic 0 .
\end{abstract}

Key words: Lie superalgebra of Weyl type, 2-cocycle

\section{$\S 1$. Introduction}

Recently there appeared a number of papers on the structure theory of infinite dimensional Lie (super)algebras and conformal (super)algebras (for example, [SXZ, SZ1-SZ3, SZZ, $\mathrm{X} 1-\mathrm{X} 5, \mathrm{Z}]$ and references cited in those papers). Among them, a class of Lie algebras of Weyl type, which is closely related to $\mathcal{W}$-infinity algebras $\mathcal{W}_{1+\infty}, \mathcal{W}_{\infty}$ and the general conformal algebras $g c_{N}$ (see, e.g., [BKV, S3, S4]), was studied in [SZ1, SZZ, Z]. In this paper, we study the 2-cohomology groups of the Lie superalgebras of Weyl type, a natural generalization of Lie algebras of Weyl type, which are closely related to $\mathcal{W}$-infinity superalgebras and general conformal superalgebras. The main result of this paper is Theorem 3.5. Since the classification of finite dimensional simple Lie superalgebras was given in [K2], the role of Lie superalgebras has become more and more important in solving problems in the quantum field theory and string theory. A cohomology theory of Lie superalgebras and Lie color algebras was developed in [ScZ1], while a general theory of cohomology of Lie conformal algebras was established in $[\mathrm{BKV}]$. The cohomology theory of Lie algebras has played important roles in the structure and representation theories of Lie algebras. It is well-known that central extensions, which are determined by 2-cohomology groups, are widely used in the structure theory and the representation theory of Lie algebras (e.g., [K1, KP, S3]). Unlike the finite

\footnotetext{
${ }^{1}$ Supported by NSF grant 10171064 of China
} 
dimensional simple Lie algebras, since the complete reducibility of modules does not hold in super case or conformal case, one may expect that, as pointed in [BKV], the cohomology theory of Lie superalgebras and conformal superalgebras, is very interesting and important, and further, one may expect that it is more difficult as is already seen even for the case of finite dimensional classical simple Lie superalgebras $\operatorname{sl}(m / n)$ [ScZ1, ScZ2].

The problem of determining the cohomology groups of general conformal algebras $g c_{N}$ remains open (see $[\mathrm{BKV}])$. Using some techniques developed in [S2] which determined 2cocycles of Lie algebras of Weyl type, a partial answer to the problem was given in [S4]. One of our motivation in this paper is to develop some results and techniques in order to be used to determine cohomology groups of the general conformal superalgebras in the future.

Now we begin with some brief definitions. Let $\mathbb{F}$ be a field of characteristic 0. For any positive integer $\ell$, an additive subgroup $\Gamma$ of the $\ell$-dimensional vector space $\mathbb{F}^{\ell}$ is called nondegenerate if $\Gamma$ contains an $\mathbb{F}$-basis of $\mathbb{F}^{\ell}$. Let $\ell_{1}, \ell_{2}, \cdots, \ell_{5}$ be five nonnegative integers such that $\ell=\sum_{p=1}^{5} \ell_{p}>0$. For convenience, we denote

$$
\ell_{i}^{\prime}=\sum_{p=1}^{i} \ell_{p}, \text { and } \overline{m, n}=\{m, m+1, \cdots, n\} \text { if } m, n \in \mathbb{Z}, m \leq n
$$

An element of $\mathbb{F}^{\ell}$ will be written as $\alpha=\left(\alpha_{1}, \ldots, \alpha_{\ell}\right)$. Take an additive subgroup $\Gamma$ of $\mathbb{F}^{\ell}$ such that

$$
\alpha=\left(\alpha_{1}, \cdots, \alpha_{\ell}\right)=\left(0, \cdots, 0, \alpha_{\ell_{1}+1}, \cdots, \alpha_{\ell_{4}^{\prime}}, 0 \cdots, 0\right) \in\{0\}^{\ell_{1}} \times \mathbb{F}^{\ell_{2}+\ell_{3}+\ell_{4}} \times\{0\}^{\ell_{5}}
$$

for all $\alpha \in \Gamma$, and such that $\Gamma$ is nondegenerate as a subgroup of $\mathbb{F}^{\ell_{2}+\ell_{3}+\ell_{4}}$. Set

$$
\vec{J}=\mathbb{Z}_{+}^{\ell_{2}^{\prime}} \times \mathbb{Z}^{\ell_{3}} \times\{0\}^{\ell_{4}} \times\{0,1\}^{\ell_{5}}
$$

For convenience, we shall always decompose a vector $\vec{k}=\left(k_{1}, \ldots, k_{\ell}\right) \in \vec{J}$ as

$$
\begin{aligned}
& \vec{k}=\vec{i}+\vec{j}, \text { where, } \\
& \vec{i}=\left(i_{1}, \ldots, i_{\ell}\right)=\left(k_{1}, \cdots, k_{\ell_{3}^{\prime}}, 0, \cdots, 0\right) \in \mathbb{Z}^{\ell_{3}^{\prime}} \times\{0\}^{\ell_{4}+\ell_{5}}, \\
& \vec{j}=\left(j_{1}, \ldots, j_{\ell}\right)=\left(0, \cdots, 0, k_{\ell_{4}^{\prime}+1}, \cdots, k_{\ell}\right) \in\{0\}^{\ell^{\prime}} \times\{0,1\}^{\ell_{5}},
\end{aligned}
$$

and we denote

$$
|\vec{k}|=\sum_{p=1}^{\ell}\left|k_{p}\right|, \quad a_{[p]}=(0, \cdots, 0, a, 0, \cdots, 0)
$$


where $a \in \mathbb{Z}$ if $p \in \overline{1, \ell_{4}^{\prime}}$, and $a \in\{0,1\}$ if $p \in \overline{\ell_{4}^{\prime}+1, \ell}$.

Let

$$
\mathcal{A}_{1}=\mathbb{F}[\Gamma]=\operatorname{span}\left\{x^{\alpha} \mid \alpha \in \Gamma\right\}
$$

be the group algebra with product $x^{\alpha} x^{\beta}=x^{\alpha+\beta}$ for $\alpha, \beta \in \Gamma$. Let

$$
\mathcal{A}_{2}=\mathbb{F}[\vec{J}]=\mathbb{F}\left[t_{1}, t_{2}, \cdots, t_{\ell_{3}^{\prime}}, s_{1}, s_{2}, \cdots, s_{\ell_{5}}\right]
$$

be the (super)polynomial algebra with the ordinary (or even) variables $t_{p}, p \in \overline{1, \ell_{3}^{\prime}}$ and the Grassmannian (or odd) variables $s_{q}, q \in \overline{1, \ell_{5}}$, namely, variables $t_{p}, s_{q}$ satisfy

$$
\left.t_{p} a=a t_{p}, \quad s_{q} s_{q^{\prime}}=-s_{q^{\prime}} s_{q} \text { (in particular, } s_{q}^{2}=0\right)
$$

for all $a \in \mathcal{A}_{2}, p \in \overline{1, \ell_{3}}, q, q^{\prime} \in \overline{1, \ell_{5}}$. Let $\mathcal{A}=\mathcal{A}_{1} \otimes \mathcal{A}_{2}$, and denote

$$
x^{\alpha, \vec{k}}=x^{\alpha} t_{1}^{i_{1}} \cdots t_{\ell_{3}^{\prime}}^{i_{\ell_{3}^{\prime}}} s_{1}^{j_{\ell^{\prime}+1}} \cdots s_{\ell_{5}}^{j_{\ell}}, \quad x^{0,0}=1, \quad t^{\vec{i}}=x^{0, \vec{i}}, \quad s^{\vec{j}}=x^{0, \vec{j}},
$$

for $\alpha \in \Gamma$ and $\vec{k} \in \vec{J}$ written as in (1.4). Then $\mathcal{A}=\mathbb{F}[\Gamma \times \vec{J}]$ is a semi-group superalgebra with basis $\left\{x^{\alpha, \vec{k}} \mid(\alpha, \vec{k}) \in \Gamma \times \vec{J}\right\}$, and the product

$$
x^{\alpha, \vec{k}} x^{\alpha^{\prime}, \overrightarrow{k^{\prime}}}=x^{\alpha, \vec{i}+\vec{j}} x^{\alpha^{\prime}, \overrightarrow{i^{\prime}}+\overrightarrow{j^{\prime}}}=(-1)^{\sum_{\ell_{4}^{\prime}<p<q \leq \ell} j_{q} j_{p}^{\prime}} x^{\alpha+\alpha^{\prime}, \vec{k}+\overrightarrow{k^{\prime}}}
$$

for $\alpha, \alpha^{\prime} \in \Gamma, \vec{k}, \vec{k}^{\prime} \in \vec{J}$, where we have used the following convention.

Convention 1.1. If an undefined notion appears in an expression, we regard it as zero; for instance, $x^{\alpha, \vec{k}}=0$ if $k_{p} \geq 2$ for some $p \in \overline{\ell_{4}^{\prime}, \ell}$ since in this case $\vec{k} \notin \vec{J}$.

Let $\mathbb{Z}_{2}=\mathbb{Z} / 2 \mathbb{Z}=\{\overline{0}, \overline{1}\}$. Then $\mathcal{A}=\mathcal{A}_{\overline{0}}+\mathcal{A}_{\overline{1}}$ is a $\mathbb{Z}_{2}$-graded supercommutative superalgebra with the gradation spaces given by

$$
\mathcal{A}_{\overline{0}}=\operatorname{span}\left\{x^{\alpha, \vec{i}+\vec{j}}|| \vec{j} \mid \text { is even }\right\}, \quad \mathcal{A}_{\overline{1}}=\operatorname{span}\left\{x^{\alpha, \vec{i}+\vec{j}}|| \vec{j} \mid \text { is odd }\right\} .
$$

Define the linear transformations $\left\{\partial_{1}^{-}, \partial_{2}^{-}, \cdots, \partial_{\ell_{3}^{\prime}}^{-}, \partial_{\ell_{3}^{\prime}+1}^{+}, \cdots, \partial_{\ell_{4}^{\prime}}^{+}, \partial_{\ell_{4}^{\prime}+1}^{-}, \cdots, \partial_{\ell}^{-}\right\}$on $\mathcal{A}$ by

$$
\partial_{p}^{-}\left(x^{\alpha, \vec{k}}\right)=k_{p} x^{\alpha, k-\overrightarrow{1}_{[p]}}, \quad \partial_{p^{\prime}}^{+}\left(x^{\alpha, \vec{k}}\right)=\alpha_{p^{\prime}} x^{\alpha, \vec{k}}, \quad \partial_{q}^{-}\left(x^{\alpha, \vec{k}}\right)=(-1)^{\sum_{r=\ell_{4}^{\prime}+1}^{q-1} k_{r}} k_{q} x^{\alpha, \vec{k}-1_{[q]}}
$$

for $p \in \overline{1, \ell_{3}^{\prime}}, p^{\prime} \in \overline{\ell_{1}+1, \ell_{4}^{\prime}}, q \in \overline{\ell_{4}^{\prime}+1, \ell}$. We call the operators $\partial_{p}^{-}$down-grading operators, and the operators $\partial_{p^{\prime}}^{+}$grading operators. Set

$$
\partial_{q}=\partial_{q}^{-}, \quad \partial_{p}=\partial_{p}^{-}+\partial_{p}^{+}, \quad \partial_{p^{\prime}}=\partial_{p^{\prime}}^{+} \quad \text { and } \quad \check{\partial}_{r}=\partial_{\ell_{4}+r},
$$


for $q \in \overline{1, \ell_{1}} \cup \overline{\ell_{4}^{\prime}+1, \ell}, p \in \overline{\ell_{1}+1, \ell_{3}^{\prime}}, p^{\prime} \in \overline{\ell_{3}^{\prime}+1, \ell_{4}^{\prime}}$ and $r \in \overline{1, \ell_{5}}$. Then $\check{\partial}_{r}, r \in \overline{1, \ell_{5}}$ are the odd (or Grassmannian) derivations satisfying $\check{\partial}_{r}^{2}=0$.

Denote $\mathcal{D}=\sum_{p=1}^{\ell} \mathbb{F} \partial_{p}$. Let $\mathbb{F}[\mathcal{D}]$ be the (super) polynomial algebra of $\mathcal{D}$ with basis

$$
\left\{\partial^{\mu}=\prod_{p=1}^{\ell} \partial_{p}^{\mu_{p}} \mid \mu=\left(\mu_{1}, \ldots, \mu_{\ell}\right) \in \vec{K}\right\}, \quad \text { where } \quad \vec{K}=\mathbb{Z}_{+}^{\ell_{4}^{\prime}} \times\{0,1\}^{\ell_{5}}
$$

and where $\partial^{\mu}=1$ if $\mu=0$. Then the vector space

$$
\mathcal{W}=\mathcal{W}\left(\ell_{1}, \cdots, \ell_{5}, \Gamma\right)=\mathcal{A} \otimes \mathbb{F}[\mathcal{D}]=\operatorname{span}\left\{x^{\alpha, \vec{k}} \partial^{\mu} \mid(\alpha, \vec{k}, \mu) \in \Gamma \times \vec{J} \times \vec{K}\right\}
$$

becomes a $\mathbb{Z}_{2}$-graded associative superalgebra, called a superalgebra of generalized Weyl type, under the operations (cf. [SZ1, SZZ])

$$
u \partial^{\mu} \cdot v \partial^{\nu}=u \sum_{\lambda \in \vec{K}}\left(\begin{array}{c}
\mu \\
\lambda
\end{array}\right)(-1)^{g(v) g(\mu-\lambda)+\sum_{\ell_{4}^{\prime}<p<q \leq \ell}\left(\mu_{q}-\lambda_{q}\right) \nu_{p}} \partial^{\lambda}(v) \partial^{\mu+\nu-\lambda},
$$

where $v$ is a homogeneous element of $\mathcal{A}$ with degree $g(v) \in \mathbb{Z}_{2}$ (the gradation of the elements in $\mathcal{W}$ is defined by (1.11) and by $g\left(\partial_{p}\right)=\overline{0}$ if $p \leq \ell_{4}^{\prime}$ and $g\left(\partial_{p}\right)=\overline{1}$ otherwise), and in general $g(\mu)=\sum_{p=\ell_{4}^{\prime}+1}^{\ell} \mu_{p}$ for $\mu \in \vec{K}$, and $\left(\begin{array}{c}\mu \\ \lambda\end{array}\right)=\prod_{p=1}^{\ell}\left(\begin{array}{c}\mu_{p} \\ \lambda_{p}\end{array}\right)$ (here $\left(\begin{array}{c}j \\ i\end{array}\right)$ is defined to be

$j(j-1) \cdots(j-i+1) / i$ ! if $i \geq 0$ or 0 otherwise $)$, and $\partial^{\lambda}(v)=\partial_{1}^{\lambda_{1}}\left(\partial_{2}^{\lambda_{2}}\left(\cdots\left(\partial_{\ell}^{\lambda_{\ell}}(v)\right)\right)\right)$. Here we have again used Convention 1.1; for instance, $\partial^{2^{\left[\ell_{4}^{\prime}+1\right]}}=0$ since $2_{\left[\ell_{4}^{\prime}+1\right]} \notin \vec{K}$. We shall ALWAYS omit the product notion "." when the context is clear.

Under the usual bracket, the superalgebra $\mathcal{W}$ induces a Lie superalgebra, also denoted by $\mathcal{W}$ and called a Lie superalgebra of generalized Weyl type, which is central simple in the sense that $[\mathcal{W}, \mathcal{W}] / \mathbb{F}$ is simple (see $[\mathrm{SZZ}])$.

\section{$\S 2 . \quad$ Preliminaries}

Since we shall be mainly interested in infinite dimensional cases, we assume $\ell_{4}^{\prime} \neq 0$. Choose a basis of $\mathcal{W}$ to be $B=\left\{x^{\alpha, \vec{k}} \partial^{\mu} \mid(\alpha, \vec{k}, \mu) \in \Gamma \times \vec{J} \times \vec{K}\right\}$. Fix an element

$$
\tau \in \Gamma \text { such that } \tau_{p} \neq 0 \text { for all } p \in \overline{\ell_{1}+1, \ell_{4}^{\prime}} \text {. }
$$

For $\mu=\left(\mu_{1}, \cdots, \mu_{\ell}\right) \in \vec{K}$, we define the level of $\mu$, by $|\mu|=\sum_{p=1}^{\ell} \mu_{p}$, and define a total order on $\vec{K}$ by

$$
\mu<\mu^{\prime} \Leftrightarrow|\mu|<\left|\mu^{\prime}\right| \text {, or }|\mu|=\left|\mu^{\prime}\right|, \exists p \text { such that } \mu_{q}=\mu_{q}^{\prime}(q<p) \text { and } \mu_{p}<\mu_{p}^{\prime} \text {. }
$$


Recall that a 2 -cocycle on Lie superalgebra $\mathcal{W}$ is an $\mathbb{F}$-bilinear function $\psi: \mathcal{W} \times \mathcal{W} \rightarrow \mathbb{F}$, satisfying the following conditions

$$
\begin{aligned}
& \psi\left(u_{1}, u_{2}\right)=-(-1)^{g\left(u_{1}\right) g\left(u_{2}\right)} \psi\left(u_{1}, u_{2}\right) \quad \text { (super-skew-symmetry), } \\
& \psi\left(u_{1},\left[u_{2}, u_{3}\right]\right)=\psi\left(\left[u_{1}, u_{2}\right], u_{3}\right)+(-1)^{g\left(u_{1}\right) g\left(u_{2}\right)} \psi\left(u_{2},\left[u_{1}, u_{2}\right]\right) \quad \text { (super-Jacobi identity), }
\end{aligned}
$$

for $u_{1}, u_{2}, u_{3} \in \mathcal{W}$ (we shall always assume an element in $\mathcal{W}$ is homogeneous). The vector space of 2-cocycles on $\mathcal{W}$ is denoted by $C^{2}(\mathcal{W}, \mathbb{F})$. A 2-cocycle $\psi$ is called 2-coboundary or trivial if there exists an $\mathbb{F}$-linear function $f$ on $\mathcal{W}$ such that $\psi=\psi_{f}$, where

$$
\psi_{f}\left(u_{1}, u_{2}\right)=f\left(\left[u_{1}, u_{2}\right]\right) \text { for } u_{1}, u_{2} \in \mathcal{W} \text {. }
$$

Denote the vector space of all 2-coboundaries by $B^{2}(\mathcal{W}, \mathbb{F})$. Two 2-cocycles $\psi, \phi$ are equivalent if $\phi-\psi$ is trivial, the quotient space

$$
H^{2}(\mathcal{W}, \mathbb{F})=C^{2}(\mathcal{W}, \mathbb{F}) / B^{2}(\mathcal{W}, \mathbb{F})
$$

is called the 2-cohomology group of $\mathcal{W}$.

The first preliminary result in obtaining our main theorem is the following rather technical lemma.

Lemma 2.1. Let $\psi$ be a 2-cocycle on Lie superalgebra $\mathcal{W}$, then there exists a 2-cocycle $\varphi$ equivalent to $\psi$ such that

$$
\begin{aligned}
\varphi\left(t_{p} \partial_{p}, x^{\alpha, \vec{k}} \partial^{\mu}\right)=0 \quad \text { if } \quad p \in \overline{1, \ell_{3}^{\prime},} \\
\varphi\left(\partial_{p}, x^{\alpha, \vec{k}} \partial^{\mu}\right)=0 \quad \text { if } \quad p \in \overline{1, \ell}, \\
\varphi\left(s_{p} \check{\partial}_{p}, x^{\alpha, \vec{k}} \partial^{\mu}\right)=0 \quad \text { if } \quad p \in \overline{1, \ell_{5}},
\end{aligned}
$$

for $(\alpha, \vec{k}, \mu) \in \Gamma \times \vec{J} \times \vec{K}$ (cf. notations (1.9) and (1.13)).

Proof. Define an $\mathbb{F}$-linear function $f: \mathcal{W} \rightarrow \mathbb{F}$ as follows: For $x^{\alpha, \vec{k}} \partial^{\mu} \in B$ with $\alpha \neq 0$, let $q \in \overline{\ell_{1}+1, \ell_{4}^{\prime}}$ be the minimal index such that $\alpha_{q} \neq 0$, we define $f\left(x^{\alpha, \vec{k}} \partial^{\mu}\right)$, inductively on $\left|k_{q}\right|$, by

$$
f\left(x^{\alpha, \vec{k}} \partial^{\mu}\right)= \begin{cases}\alpha_{q}^{-1}\left(\psi\left(\partial_{q}, x^{\alpha, \vec{k}} \partial^{\mu}\right)-k_{q} f\left(x^{\alpha, \vec{k}-1_{[q]}} \partial^{\mu}\right)\right) & \text { if } k_{q} \geq 0 \\ -\left(1+\mu_{q}\right)^{-1}\left(\psi\left(t_{q} \partial_{q}, x^{\alpha, \vec{k}} \partial^{\mu}\right)-\alpha_{q} f\left(x^{\alpha, \vec{k}+1_{[q]}} \partial^{\mu}\right)\right) & \text { if } k_{q}=-1 \\ \left(k_{q}+1\right)^{-1}\left(\psi\left(\partial_{q}, x^{\alpha, \vec{k}+1_{[1 q]}} \partial^{\mu}\right)-\alpha_{q} f\left(x^{\alpha, \vec{k}+1_{[q]}} \partial^{\mu}\right)\right) & \text { if } k_{q} \leq-2\end{cases}
$$


Note that $\partial_{q} \in \mathcal{W}_{\overline{0}}$ and $\left[\partial_{q}, x^{\alpha, \vec{k}} \partial^{\mu}\right]=\alpha_{q} x^{\alpha, \vec{k}} \partial^{\mu}+k_{q} x^{\alpha, \vec{k}-1_{[q]}} \partial^{\mu}$. If $k_{q} \geq 1$, then $\left(\vec{k}-1_{[q]}\right) \in \vec{J}$, so,

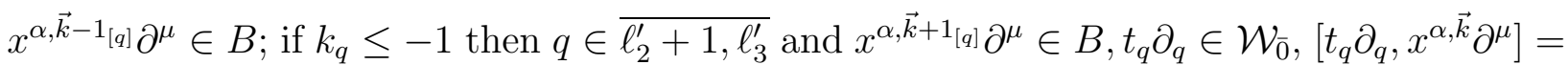
$\alpha_{q} x^{\alpha, \vec{k}+1_{[q]}} \partial^{\mu}+\left(k_{q}-\mu_{q}\right) x^{\alpha, \vec{k}} \partial^{\mu}$. Thus the right-hand side of (2.8) makes sense in all cases.

For $t^{\vec{k}} \partial^{\mu} \in B$ with $\vec{k}=\vec{i}+\vec{j}$ and $\vec{i} \neq 0$, let $r$ be the minimal index such that $i_{r} \neq 0$, we define

$$
f\left(t^{\vec{k}} \partial^{\mu}\right)= \begin{cases}\left(i_{r}-\mu_{r}\right)^{-1} \psi\left(t_{r} \partial_{r}, t^{\vec{k}} \partial^{\mu}\right) & \text { if } i_{r} \neq \mu_{r}, \\ \left(i_{r}+1\right)^{-1} \psi\left(\partial_{r}, t^{\vec{k}+1_{[r]}} \partial^{\mu}\right) & \text { if } i_{r}=\mu_{r} .\end{cases}
$$

Note that in (2.9) since $i_{r} \neq 0$, we have $r \leq \ell_{3}^{\prime}$ and $t_{r} \partial_{r} \in \mathcal{W}_{\overline{0}}$, so the right-hand side of (2.9) makes sense.

For $s^{\vec{j}} \partial^{\mu} \in B$ with $\vec{j} \neq 0$, let $r^{\prime} \in \overline{\ell_{4}^{\prime}+1, \ell}$ be the minimal index such that $j_{r^{\prime}} \neq 0$ (i.e., $\left.j_{r^{\prime}}=1\right)$, we define

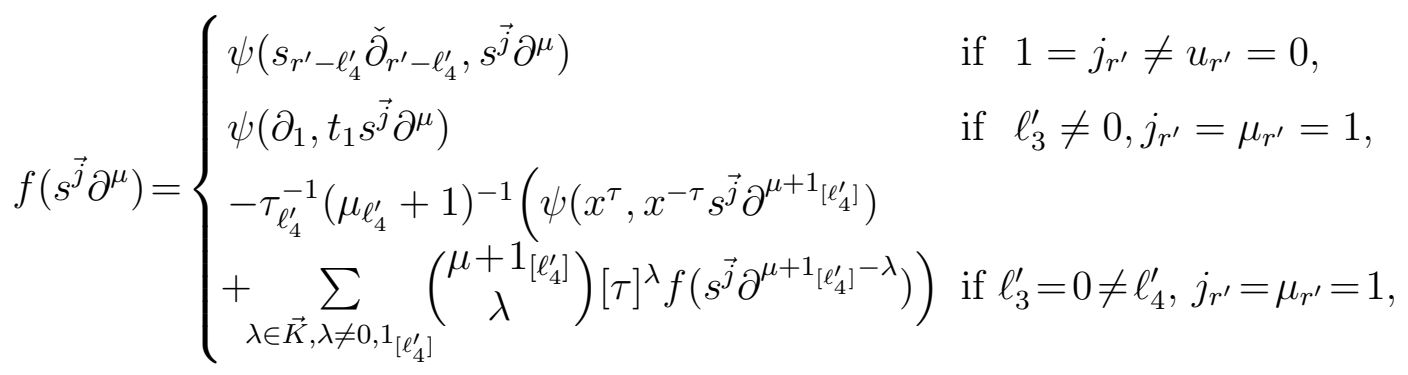

where in general we denote

$$
[\alpha]^{\lambda}=\prod_{p=1}^{\ell_{4}^{\prime}} \alpha_{p}^{\lambda_{p}} \quad \text { for } \quad \alpha \in \Gamma, \lambda \in \vec{K} .
$$

Note that $\left[s_{r^{\prime}-\ell_{4}^{\prime}} \check{\partial}_{r^{\prime}}-\ell_{4}^{\prime}, s^{\vec{j}} \partial^{\mu}\right]=s^{\vec{j}} \partial^{\mu}$ if $1=j_{r^{\prime}} \neq \mu_{r^{\prime}}=0$, and $\left[\partial_{1}, t_{1} s^{\vec{j}} \partial^{\mu}\right]=s^{\vec{j}} \partial^{\mu}$ if $\ell_{3}^{\prime} \neq 0$, and

$$
\left[x^{\tau}, x^{-\tau} s^{\vec{j}} \partial^{\left.\mu+1_{\left[\ell_{4}^{\prime}\right]}\right]}\right]=-\tau_{\ell_{4}^{\prime}}\left(\mu_{\ell_{4}^{\prime}}+1\right) s^{\vec{j}} \partial^{\mu}-\sum_{\lambda \in \vec{K}, \lambda \neq 0,1_{\left[\ell_{4}^{\prime}\right]}} x^{-\tau} s^{\vec{j}}\left(\begin{array}{c}
\mu+1_{\left[\ell_{4}^{\prime}\right]} \\
\lambda
\end{array}\right) \partial^{\lambda}\left(x^{\tau}\right) \partial^{\mu+1_{\left[\ell_{4}^{\prime}\right]}-\lambda}
$$

if $\ell_{3}^{\prime}=0 \neq \ell_{4}^{\prime}$. We define $f\left(s^{\vec{j}} \partial^{\mu}\right)$ by induction on $\mu$ with respect to the order defined in (2.2).

Finally for $\partial^{\mu} \in B$ with $\mu \in \vec{K}$, if $\ell_{3}^{\prime} \neq 0$, we define

$$
f\left(\partial^{\mu}\right)=\psi\left(\partial_{1}, t_{1} \partial^{\mu}\right)
$$

and if $\ell_{3}^{\prime}=0 \neq \ell_{4}^{\prime}$, we define (cf. (2.11) and (2.12))

$$
f\left(\partial^{\mu}\right)=-\left(\tau_{\ell_{4}^{\prime}}\left(\mu_{\ell_{4}^{\prime}}+1\right)\right)^{-1}\left(\psi\left(x^{\tau}, x^{-\tau} \partial^{\left.\mu+1_{\left[\ell_{4}^{\prime}\right]}\right]}\right)+\sum_{\lambda \in \vec{K}, \lambda \neq 0,1_{\left[\ell_{4}^{\prime}\right]}}\left(\begin{array}{c}
\mu+1_{\left[\ell_{4}^{\prime}\right]} \\
\lambda
\end{array}\right)[\tau]^{\lambda} f\left(\partial^{\mu+1_{\left[\ell_{4}^{\prime}\right]}-\lambda}\right)\right)
$$


by induction on the order of $\mu$.

Now set $\varphi=\psi-\psi_{f}$. For $v=x^{\alpha, \vec{k}} \partial^{\mu} \in B$, we define

$$
\begin{cases}q=q_{v}=\min \left\{q \in \overline{\ell_{1}+1, \ell_{4}^{\prime}} \mid \alpha_{q} \neq 0\right\} & \text { if } \quad \alpha \neq 0, \\ r=r_{v}=\min \left\{r \in \overline{1, \ell_{3}^{\prime}} \mid i_{r} \neq 0\right\} & \text { if } \quad \vec{i} \neq 0, \\ r^{\prime}=r_{v}^{\prime}=\min \left\{r^{\prime} \in \overline{\ell_{4}^{\prime}+1, \ell} \mid j_{r^{\prime}} \neq 0\right\} & \text { if } \quad \vec{j} \neq 0,\end{cases}
$$

(when there is confusion, we add subscript $v$ to the notation). Then by (2.8)-(2.14), we have

$$
\begin{aligned}
& \varphi\left(\partial_{q}, x^{\alpha, \vec{k}} \partial^{\mu}\right)=0 \quad \text { if } \alpha \neq 0, \\
& \varphi\left(t_{q} \partial_{q}, x^{\alpha, \vec{k}} \partial^{\mu}\right)=0 \quad \text { if } \alpha \neq 0, i_{q}=-1 \text {, } \\
& \varphi\left(t_{r} \partial_{r}, t^{\vec{i}} \partial^{\mu}\right)=0 \quad \text { if } i_{r} \neq \mu_{r} \\
& \varphi\left(\partial_{r}, t^{\vec{k}} \partial^{\mu}\right)=0 \quad \text { if } i_{r} \geq 2, \mu_{r}=i_{r}-1 \text {, or } r=1, \vec{i}=1_{[1]} \text {, } \\
& \varphi\left(s_{r^{\prime}-\ell_{4}^{\prime}} \check{\partial}_{r^{\prime}-\ell_{4}^{\prime}}, s^{\vec{j}} \partial^{\mu}\right)=0 \quad \text { if } 1=j_{r^{\prime}} \neq \mu_{r^{\prime}}=0 \text {, } \\
& \varphi\left(\partial_{1}, t_{1} s^{\vec{j}} \partial^{\mu}\right)=0 \quad \text { if } \ell_{3}^{\prime} \neq 0, j_{r^{\prime}}=\mu_{r^{\prime}}=1, \\
& \varphi\left(x^{\tau}, x^{-\tau} s^{\vec{j}} \partial^{\mu+1}{ }_{\left[\ell_{4}^{\prime}\right]}\right)=0 \quad \text { if } \ell_{3}^{\prime}=0 \neq \ell_{4}^{\prime}, j_{r^{\prime}}=\mu_{r^{\prime}}=1 \text {, } \\
& \varphi\left(x^{\tau}, x^{-\tau} \partial^{\mu+1}\left[\ell_{4}^{\prime}\right]\right)=0 \quad \text { if } \ell_{3}^{\prime}=0 \neq \ell_{4}^{\prime} .
\end{aligned}
$$

Now we prove the lemma in 4 cases.

Case 1: $\alpha \neq 0$. Let $q$ be as in (2.15), by (2.16) and (2.17) we obtain

$$
\begin{aligned}
0 & =\varphi\left(\partial_{q}, \alpha_{p} x^{\alpha, \vec{k}+1_{[p]}} \partial^{\mu}+\left(k_{p}-\mu_{p}\right) x^{\alpha, \vec{k}} \partial^{\mu}\right)=\varphi\left(\partial_{q},\left[t_{p} \partial_{p}, x^{\alpha, \vec{k}} \partial^{\mu}\right]\right) \\
& =\varphi\left(\left[\partial_{q}, t_{p} \partial_{p}\right], x^{\alpha, \vec{k}} \partial^{\mu}\right)+\varphi\left(t_{p} \partial_{p},\left[\partial_{q}, x^{\alpha, \vec{k}} \partial^{\mu}\right]\right) \\
& =\delta_{p, q} \varphi\left(\partial_{p}, x^{\alpha, \vec{k}} \partial^{\mu}\right)+\varphi\left(t_{p} \partial_{p}, \alpha_{q} x^{\alpha, \vec{k}} \partial^{\mu}+i_{q} x^{\alpha, \vec{k}-1_{[q]}} \partial^{\mu}\right) \\
& =\alpha_{q} \varphi\left(t_{p} \partial_{p}, x^{\alpha, \vec{k}} \partial^{\mu}\right)+i_{q} \varphi\left(t_{p} \partial_{p}, x^{\alpha, \vec{k}-1_{[q]}} \partial^{\mu}\right) \quad \text { for } p \in \overline{1, \ell_{3}^{\prime}} .
\end{aligned}
$$

We also have

$$
\begin{aligned}
0 & =\varphi\left(\partial_{q}, \alpha_{p} x^{\alpha, \vec{k}} \partial^{\mu} \pm i_{p} x^{\alpha, \vec{k}-1_{[p]}} \partial^{\mu}\right)=\varphi\left(\partial_{q},\left[\partial_{p}, x^{\alpha, \vec{k}} \partial^{\mu}\right]\right) \\
& =\alpha_{q} \varphi\left(\partial_{p}, x^{\alpha, \vec{k}} \partial^{\mu}\right)+i_{q} \varphi\left(\partial_{p}, x^{\alpha, \vec{k}-1_{[q]}} \partial^{\mu}\right) \quad \text { for } p \in \overline{1, \ell}
\end{aligned}
$$

(note that when $p \in \overline{\ell_{4}^{\prime}+1, \ell}, \partial_{p}$ is an odd derivation, so it may produce a minus sign when applying it to $\left.x^{\alpha, \vec{k}}\right)$. If $i_{q} \geq 0$, then (2.5) and (2.6) follow from (2.24), (2.25) and induction 
on $i_{q}$. If $i_{q}=-1, p=q$, then (2.5) and (2.6) follow from (2.16) and (2.17). Assume $i_{q}=-1, p \neq q$. Using $(2.17)$ we have

$$
\begin{aligned}
& 0=\varphi\left(t_{q} \partial_{q},\left[t_{p} \partial_{p}, x^{\alpha, \vec{k}} \partial^{\mu}\right]\right)=\varphi\left(t_{p} \partial_{p},\left[t_{q} \partial_{q}, x^{\alpha, \vec{k}} \partial^{\mu}\right]\right) \\
& =\varphi\left(t_{p} \partial_{p}, \alpha_{q} x^{\alpha, \vec{k}+1_{[q]}} \partial^{\mu}+i_{q} x^{\alpha, \vec{k}} \partial^{\mu}-\mu_{q} x^{\alpha, \vec{k}} \partial^{\mu}\right)=-\left(1+\mu_{q}\right) \varphi\left(t_{p} \partial_{p}, x^{\alpha, \vec{k}} \partial^{\mu}\right) \text {, }
\end{aligned}
$$

which implies (2.5), where the last equality of (2.26) follows from the fact that (2.5) holds when $i_{q} \geq 0$. Using (2.24), the proof of (2.5) can be completed by induction on $-i_{q}$ when $i_{q} \leq-2$. To complete the proof of (2.6), first suppose $p \in \overline{1, \ell_{4}^{\prime}}$. If $i_{q}=-1$, then

$$
\begin{aligned}
0 & =\varphi\left(t_{q} \partial_{q},\left[\partial_{p}, x^{\alpha, \vec{k}} \partial^{\mu}\right]\right) \\
& =\varphi\left(\left[t_{q} \partial_{q}, \partial_{p}\right], x^{\alpha, \vec{k}} \partial^{\mu}\right)+\varphi\left(\partial_{p},\left[t_{q} \partial_{q}, x^{\alpha, \vec{k}} \partial^{\mu}\right]\right) \\
& =-\delta_{p, q} \varphi\left(\partial_{p}, x^{\alpha, \vec{k}} \partial^{\mu}\right)+\alpha_{q} \varphi\left(\partial_{p}, x^{\alpha, \vec{k}+1}\left[{ }_{[q]} \partial^{\mu}\right)-\left(1+\mu_{q}\right) \varphi\left(\partial_{p}, x^{\alpha, \vec{k}} \partial^{\mu}\right)\right. \\
& =-\left(1+\delta_{p, q}+\mu_{q}\right) \varphi\left(\partial_{p}, x^{\alpha, \vec{k}} \partial^{\mu}\right),
\end{aligned}
$$

which implies (2.6), where the last equality of (2.27) follows from the fact that (2.6) holds when $i_{q} \geq 0$. If $i_{q} \leq-2$, (2.6) is obtained from (2.25) by induction on $-i_{q}$. Next suppose $p \in \overline{\ell_{4}^{\prime}+1, \ell}$. If $i_{q}=-1$ then from (2.5) we have (noting that $p \neq q$ in this case)

$$
\begin{aligned}
0 & =\varphi\left(t_{q} \partial_{q},\left[\partial_{p}, x^{\alpha, \vec{k}} \partial^{\mu}\right]\right)=\varphi\left(\left[t_{q} \partial_{q}, \partial_{p}\right], x^{\alpha, \vec{k}} \partial^{\mu}\right)+\varphi\left(\partial_{p},\left[t_{q} \partial_{q}, x^{\alpha, \vec{k}} \partial^{\mu}\right]\right) \\
& =\alpha_{q} \varphi\left(\partial_{p}, x^{\alpha, \vec{k}+1_{[q]}} \partial^{\mu}\right)-\left(1+\mu_{q}\right) \varphi\left(\partial_{p}, x^{\alpha, \vec{k}} \partial^{\mu}\right)=-\left(1+\mu_{q}\right) \varphi\left(\partial_{p}, x^{\alpha, \vec{k}} \partial^{\mu}\right),
\end{aligned}
$$

which implies (2.6). The proof of (2.6) is completed by (2.25) and induction on $-i_{q}$ when $i_{q} \leq-2$. The proof of $(2.7)$ is similar to that of $(2.5)$.

Case 2: $\alpha=0, \vec{i} \neq 0$ (which implies $\ell_{3}^{\prime} \geq 1$ ). Let $r$ be as in (2.15). First we prove (2.5). If $i_{r} \neq \mu_{r}$, then by $(2.18)$ we have

$$
\begin{aligned}
0 & =\varphi\left(t_{r} \partial_{r},\left[t_{p} \partial_{p}, t^{\vec{k}} \partial^{\mu}\right]\right)=\varphi\left(\left[t_{r} \partial_{r}, t_{p} \partial_{p}\right], t^{\vec{k}} \partial^{\mu}\right)+\varphi\left(t_{p} \partial_{p},\left[t_{r} \partial_{r}, t^{\vec{k}} \partial^{\mu}\right]\right) \\
& =\left(i_{r}-\mu_{r}\right) \varphi\left(t_{p} \partial_{p}, t^{\vec{k}} \partial^{\mu}\right) \quad\left(\text { noting that } r, p \in \overline{1, \ell_{3}^{\prime}}\right)
\end{aligned}
$$

which implies (2.5). Assume $i_{r}=\mu_{r}$. Then we have $i_{r} \geq 1$ (cf. (2.15)), and

$$
\begin{aligned}
\varphi\left(t_{p} \partial_{p}, t^{\vec{k}} \partial^{\mu}\right) & =\left(1+i_{r}\right)^{-1} \varphi\left(t_{p} \partial_{p},\left[\partial_{r}, t^{\vec{k}+1_{[r]}} \partial^{\mu}\right]\right) \\
& =\left(1+i_{r}\right)^{-1}\left(-\delta_{r, p}+i_{p}+\delta_{p, r}-\mu_{p}\right) \varphi\left(\partial_{r}, t^{\vec{k}+1_{[r]}} \partial^{\mu}\right)=0
\end{aligned}
$$


which gives (2.5), where the last equality follows from (2.19) by noting that the condition of (2.19) is satisfied by $t^{\vec{k}+1_{[r]}} \partial^{\mu}$ since $i_{r}+1=\mu_{r}+1 \geq 2$. Similarly we have (2.7). Next consider (2.6). First suppose $p \in \overline{1, \ell_{4}^{\prime}}$. Since

$$
t^{\vec{k}} \partial^{\mu}= \begin{cases}\left(i_{1}+1\right)^{-1}\left[\partial_{1}, t^{\vec{k}+1_{[1]}} \partial^{\mu}\right] & \text { if } i_{1} \neq-1 \\ -\left(1+\mu_{1}\right)^{-1}\left[t_{1} \partial_{1}, t^{\vec{k}} \partial^{\mu}\right] & \text { if } i_{1}=-1\end{cases}
$$

we have

$$
\varphi\left(\partial_{p}, t^{\vec{k}} \partial^{\mu}\right)= \begin{cases}\left(i_{1}+1\right)^{-1}\left(i_{p}+\delta_{p, 1}\right) \varphi\left(\partial_{1}, t^{\vec{k}+1_{[1]}-1_{[p]}} \partial^{\mu}\right) & \text { if } i_{1} \neq-1 \\ -\left(1+\mu_{1}\right)^{-1} \delta_{p, 1} \varphi\left(\partial_{1}, t^{\vec{k}} \partial^{\mu}\right) & \text { if } i_{1}=-1\end{cases}
$$

where the second case is obtained from the super-Jacobi identity and (2.5). So the proof of (2.6) is reduced the case $p=1$. Using (2.5) we have

$$
0=\varphi\left(t_{1} \partial_{1},\left[\partial_{1}, t^{\vec{k}} \partial^{\mu}\right]\right)=\left(-1+i_{1}-\mu_{1}\right) \varphi\left(\partial_{1}, t^{\vec{k}} \partial^{\mu}\right)
$$

Thus it remains to consider the case $i_{1}=\mu_{1}+1$. The result follows from (2.19) if $\mu_{1} \geq 1$ or $\vec{i}=1_{[1]}$. Thus assume $\mu_{1}=0$ and $\vec{i} \neq 1_{[1]}$. Then $i_{q^{\prime}} \neq 0$ for some $q^{\prime} \in \overline{2, \ell_{3}^{\prime}}$ and we denote $q^{\prime}$ to be the minimal index with $i_{q^{\prime}} \neq 0$. If $i_{q^{\prime}} \neq \mu_{q^{\prime}}$, then by $(2.5)$ we have

$$
0=\varphi\left(t_{q^{\prime}} \partial_{q^{\prime}},\left[\partial_{1}, t^{\vec{k}} \partial^{\mu}\right]\right)=\left(i_{q^{\prime}}-\mu_{q^{\prime}}\right) \varphi\left(\partial_{1}, t^{\vec{k}} \partial^{\mu}\right)
$$

and if $i_{q^{\prime}}=\mu_{q^{\prime}}$ then

$$
\varphi\left(\partial_{1}, t^{\vec{k}} \partial^{\mu}\right)=\left(i_{q^{\prime}}+1\right)^{-1} \varphi\left(\partial_{1},\left[\partial_{q^{\prime}}, t^{\vec{k}+1_{\left[q^{\prime}\right]}} \partial^{\mu}\right]\right)=\left(i_{q^{\prime}}+1\right)^{-1} \varphi\left(\partial_{q^{\prime}}, t^{\vec{k}-1_{[1]}+1_{\left[q^{\prime}\right]}} \partial^{\mu}\right)=0
$$

where the last equality follows from (2.19) by noting that $q^{\prime}$ is precisely the number $r_{v}$ defined in $(2.15)$ for $v=t^{\vec{k}-1_{[1]}+1_{\left[q^{\prime}\right]}} \partial^{\mu}$. Finally assume $p \in \overline{\ell_{4}^{\prime}+1, \ell}$. From the result above we have

$$
0=\varphi\left(\partial_{1},\left[\partial_{p}, t^{\vec{k}+1_{[1]}} \partial^{\mu}\right]\right)=\varphi\left(\partial_{p},\left[\partial_{1}, t^{\vec{k}+1_{[1]}} \partial^{\mu}\right]\right)=\left(i_{1}+1\right) \varphi\left(\partial_{p}, t^{\vec{k}} \partial^{\mu}\right)
$$

which implies $(2.6)$ if $i_{1} \neq-1$. If $i_{1}=-1$, then (2.5) gives

$$
0=\varphi\left(t_{1} \partial_{1},\left[\partial_{p}, t^{\vec{k}} \partial^{\mu}\right]\right)=\varphi\left(\partial_{p},\left[t_{1} \partial_{1}, t^{\vec{k}} \partial^{\mu}\right]\right)=-\left(1+\mu_{1}\right) \varphi\left(\partial_{p}, t^{\vec{k}} \partial^{\mu}\right)
$$

which implies (2.6) since $\mu_{1} \geq 0$.

Case 3: $\alpha=0, \vec{i}=0, \vec{j} \neq 0$. Consider (2.5). For $p \in \overline{1, \ell_{3}^{\prime}}$, we have

$$
\varphi\left(t_{p} \partial_{p}, s^{\vec{j}} \partial^{\mu}\right)=\varphi\left(t_{p} \partial_{p},\left[\partial_{p}, t_{p} s^{\vec{j}} \partial^{\mu}\right]\right)=0
$$


where the last equality follows from the super-Jacobi identity and Case 2. Consider (2.7). Assume $p \in \overline{1, \ell_{5}}$. If $\ell_{3}^{\prime} \neq 0$, then similar to (2.38), we have

$$
\varphi\left(s_{p} \check{\partial}_{p}, s^{\vec{j}} \partial^{\mu}\right)=\varphi\left(s_{p} \check{\partial}_{p},\left[\partial_{1}, t_{1} s^{\vec{j}} \partial^{\mu}\right]\right)=0 .
$$

Assume $\ell_{3}^{\prime}=0 \neq \ell_{4}^{\prime}$. Let $r^{\prime}$ be as in (2.15). If $\mu_{r^{\prime}}=0$, we have

$$
\varphi\left(s_{p} \check{\partial}_{p}, s^{\vec{j}} \partial^{\mu}\right)=\varphi\left(s_{p} \check{\partial}_{p},\left[s_{r^{\prime}-\ell_{4}^{\prime}} \check{\partial}_{r^{\prime}-\ell_{4}^{\prime}}, s^{\vec{j}} \partial^{\mu}\right]\right)=0 \quad \text { (cf. notations (1.9) and (1.13)), }
$$

where the last equality follows from super-Jacobi identity and (2.20). If $\mu_{r^{\prime}}=1=j_{r^{\prime}}$, we have

$$
\begin{aligned}
0 & =\varphi\left(\left[s_{p} \check{\partial}_{s}, x^{\tau}\right], x^{-\tau} s^{\vec{j}} \partial^{\mu+1_{\left[\ell_{4}^{\prime}\right]}}\right) \quad\left(\text { since }\left[s_{p} \check{\partial}_{p}, x^{\tau}\right]=0\right) \\
& =\varphi\left(s_{p} \check{\partial}_{p},\left[x^{\tau}, x^{-\tau} s^{j} \partial^{\left.\mu+1_{\left[\ell_{4}^{\prime}\right]}\right]}\right) \quad(\text { from }(2.4) \text { and }(2.22))\right. \\
& =-\sum_{0 \neq \lambda \in \vec{K}}\left(\begin{array}{c}
\mu+1_{\left[\ell_{4}^{\prime}\right]} \\
\lambda
\end{array}\right)[\tau]^{\lambda} \varphi\left(s_{p} \check{\partial}_{p}, s^{j} \partial^{\mu-\lambda+1_{\left[\ell_{4}^{\prime}\right]}}\right) \quad(\text { cf. }(2.11)),
\end{aligned}
$$

and (2.7) follows from the induction on $|\mu|$. Consider (2.6). If $p \in \overline{1, \ell_{3}^{\prime}}$, we have

$$
\begin{aligned}
0 & =\varphi\left(t_{p} \partial_{p},\left[\partial_{p}, s^{\vec{j}} \partial^{\mu}\right]\right) \quad\left(\text { since }\left[\partial_{p}, s^{\vec{j}} \partial^{\mu}\right]=0\right) \\
& =\varphi\left(\left[t_{p} \partial_{p}, \partial_{p}\right], s^{\vec{j}} \partial^{\mu}\right)+\varphi\left(\partial_{p},\left[t_{p} \partial_{p}, s^{\vec{j}} \partial^{\mu}\right]\right)=-\left(1+\mu_{p}\right) \varphi\left(\partial_{p}, s^{\vec{j}} \partial^{\mu}\right) .
\end{aligned}
$$

Assume $p \in \overline{\ell_{3}^{\prime}+1, \ell}$. If $\ell_{3}^{\prime} \geq 1$, we have

$$
\varphi\left(\partial_{p}, s^{\vec{j}} \partial^{\mu}\right)=\varphi\left(\partial_{p},\left[\partial_{1}, t_{1} s^{\vec{j}} \partial^{\mu}\right]\right)=\varphi\left(\partial_{1},\left[\partial_{p}, t_{1} s^{\vec{j}} \partial^{\mu}\right]\right)=0 \quad \text { (by Case 2). }
$$

Assume $\ell_{3}^{\prime}=0$. If there exists $p^{\prime} \in \overline{\ell_{4}^{\prime}+1, \ell} \backslash\{p\}$ such that $j_{p^{\prime}} \neq \mu_{p^{\prime}}$, then

$$
\left.\varphi\left(\partial_{p}, s^{\vec{j}} \partial^{\mu}\right)=\varphi\left(\partial_{p},\left[s_{p^{\prime}-\ell_{4}^{\prime}} \check{\partial}_{p^{\prime}-\ell_{4}^{\prime}}, s^{\vec{j}} \partial^{\mu}\right]\right)=0 \quad \text { (by (2.4) and }(2.7)\right) \text {. }
$$

Assume

$$
j_{p^{\prime}}=\mu_{p^{\prime}} \text { for all } p^{\prime} \in \overline{\ell_{4}^{\prime}+1, \ell} \backslash\{p\} .
$$

If $p=r^{\prime}$ and $1=j_{r^{\prime}}=\mu_{r^{\prime}}$, we have

$$
0=\varphi\left(s_{r^{\prime}-\ell_{4}^{\prime}} \check{\partial}_{r^{\prime}-\ell_{4}^{\prime}},\left[\partial_{p}, s^{\vec{j}} \partial^{\mu}\right]\right)=-\varphi\left(\partial_{p}, s^{\vec{j}} \partial^{\mu}\right) \quad(\text { cf. notations }(1.9) \text { and }(1.13))
$$

by super-Jacobi identity. Assume $p=r^{\prime}$ and $1=j_{r^{\prime}} \neq \mu_{r^{\prime}}=0$. Let $v=\left[\partial_{p}, x^{-\tau} s^{\vec{j}} \partial^{\mu+1_{\left[\ell_{4}^{\prime}\right]}}\right]=$ $x^{-\tau} s^{\vec{j}-1_{\left[r^{\prime}\right]}} \partial^{\mu+1_{\left[\ell_{4}^{\prime}\right]}}$. Then $v$ either has the form $x^{-\tau} \partial^{\mu+1_{\left[\ell_{4}^{\prime}\right]}}\left(\right.$ if $s^{\vec{j}}=1_{\left[r^{\prime}\right]}$ ) or $r_{v}^{\prime} \neq r^{\prime}$ satisfies $j_{r_{v}^{\prime}}=\mu_{r_{v}^{\prime}}=1$ by (2.42) and by definition (2.15); in either case, we have

$$
\varphi\left(x^{\tau}, v\right)=0 \quad(\text { by }(2.22) \text { or }(2.23)) \text {. }
$$


Thus

$$
\begin{aligned}
& 0=\varphi\left(\left[\partial_{p}, x^{\tau}\right], x^{-\tau} s^{\vec{j}} \partial^{\mu+1}\left[\ell_{4}^{\prime}\right]\right) \quad\left(\text { since }\left[\partial_{p}, x^{\tau}\right]=0\right)
\end{aligned}
$$

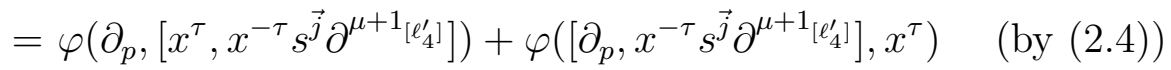

$$
\begin{aligned}
& =\varphi\left(\partial_{p},\left[x^{\tau}, x^{-\tau} s^{\vec{j}} \partial^{\left.\mu+1_{\left[\ell_{4}^{\prime}\right]}\right]}\right)\right. \\
& =-\sum_{0 \neq \lambda \in \vec{K}}\left(\begin{array}{c}
\mu+1_{\left[\ell_{4}^{\prime}\right]} \\
\lambda
\end{array}\right)[\tau]^{\lambda} \varphi\left(\partial_{p}, s^{\vec{j}} \partial^{\mu+1_{\left[\ell_{4}^{\prime}\right]}-\lambda}\right) \quad \text { (cf. (2.12)), }
\end{aligned}
$$

which gives (2.6) by induction on $\mu$, where the third equality follows from (2.43). Finally assume $p \neq r^{\prime}$. Then by (2.42) we must have $1=j_{r^{\prime}}=\mu_{r^{\prime}}$, and we still have (2.44) where now the third equality follows from (2.22).

Case 4: $\alpha=0, \vec{k}=0$. From (2.38), we have (2.5). Consider (2.6). If $p \in \overline{1, \ell_{3}^{\prime}}$, then

$$
0=\varphi\left(t^{1}{ }^{[p]} \partial_{p},\left[\partial_{p}, \partial^{\mu}\right]\right)=-\left(1+\mu_{p}\right) \varphi\left(\partial_{p}, \partial^{\mu}\right)
$$

which implies (2.6). Assume $p \in \overline{\ell_{3}^{\prime}+1, \ell}$. If $\ell_{3}^{\prime} \geq 1$, then

$$
\varphi\left(\partial_{p}, \partial^{\mu}\right)=\varphi\left(\partial_{p},\left[\partial_{1}, t^{1[1]} \partial^{\mu}\right]\right)=\varphi\left(\partial_{1},\left[\partial_{p}, t^{1_{[1]}} \partial^{\mu}\right]\right)=0
$$

If $\ell_{3}^{\prime}=0$, then

$$
0=\varphi\left(\left[\partial_{p}, x^{\tau}\right], x^{-\tau} \partial^{\mu+1_{\ell_{4}^{\prime}}}\right)=-\sum_{0 \neq \lambda \in \vec{K}}\left(\begin{array}{c}
\mu+1_{\ell_{4}^{\prime}} \\
\lambda
\end{array}\right) \prod_{q} \tau_{q}^{\lambda_{q}} \varphi\left(\partial_{p}, \partial^{\mu+1_{\left[\ell_{4}^{\prime}\right]}-\lambda}\right)
$$

by $(2.12),(2.23)$ and by noting that if $p \leq \ell_{4}^{\prime}$ then $\left[\partial_{p}, x^{\tau}\right]=\tau_{p} x^{\tau},\left[\partial_{p}, x^{-\tau} \partial^{\mu+1}{ }_{\left[\ell_{4}^{\prime}\right]}\right]=$ $-\tau_{p} x^{-\tau} \partial^{\mu+1_{\left[\ell_{4}^{\prime}\right]}}$, and if $p>\ell_{4}^{\prime}$ then $\left[\partial_{p}, x^{\tau}\right]=0,\left[\partial_{p}, x^{-\tau} \partial^{\mu+1_{\left[\ell_{4}^{\prime}\right]}}\right]=0$. Induction on $|\mu|$ gives (2.6). Finally consider (2.7). Suppose $p \in \overline{1, \ell_{5}}$. If $\ell_{3}^{\prime} \neq 0$, we have

$$
0=\varphi\left(\partial_{1},\left[s_{p} \check{\partial}_{p}, t_{1} \partial^{\mu}\right]\right)=\varphi\left(s_{p} \check{\partial}_{p},\left[\partial_{1}, t_{1} \partial^{\mu}\right]\right)=\varphi\left(s_{p} \check{\partial}_{p}, \partial^{\mu}\right)
$$

If $\ell_{3}^{\prime}=0, \ell_{4}^{\prime} \neq 0$ then by $(2.23)$ and $(2.12)$ we have

$$
\begin{aligned}
0 & =\varphi\left(\left[s_{p} \check{\partial}_{p}, x^{\tau}\right], x^{-\tau} \partial^{\mu+1_{\left[\ell_{4}^{\prime}\right]}}\right)=\varphi\left(s_{p} \check{\partial}_{p},\left[x^{\tau}, x^{-\tau} \partial^{\left.\mu+1_{\left[\ell_{4}^{\prime}\right]}\right]}\right)+\varphi\left(x^{\tau},\left[s_{p} \check{\partial}_{p}, x^{-\tau} \partial^{\left.\mu+1_{\left[\ell_{4}^{\prime}\right]}\right]}\right)\right.\right. \\
& =\sum_{0 \neq \lambda \in \vec{K}}\left(\begin{array}{c}
\mu+1_{\left[\ell_{4}^{\prime}\right]} \\
\lambda
\end{array}\right)[\tau]^{\lambda} \varphi\left(s_{p} \check{\partial}_{p}, \partial^{\mu-\lambda+1_{\left[\ell_{4}^{\prime}\right]}}\right),
\end{aligned}
$$

which gives the result by induction on $|\mu|$. This completes the proof of the lemma. 


\section{§3. Main results}

Recall that we assume $\ell_{4}^{\prime}>0$. Denote

$$
\mathcal{W}_{0}=\operatorname{span}\left\{x^{\alpha, \vec{i}} \partial^{\mu} \mid(\alpha, \vec{i}, \mu) \in \Gamma \times \vec{J}^{\prime} \times \vec{K}^{\prime}\right\}, \quad \vec{J}^{\prime}=\mathbb{Z}_{+}^{\ell_{2}^{\prime}} \times \mathbb{Z}^{\ell_{3}} \times\{0\}^{\ell_{4}+\ell_{5}}, \quad \vec{K}^{\prime}=\mathbb{Z}_{+}^{\ell_{4}^{\prime}} \times\{0\}^{\ell_{5}}
$$

(cf. (1.3) and (1.14)). Then $\mathcal{W}_{0}$ is a Lie algebra of Weyl type whose 2-cocycles were considered in [S2] (cf. also [L], [LW], [S1]). The following result can be found in [S2].

Theorem 3.1. (1) If $\ell_{4}^{\prime}=\ell_{4}=1$, then $H^{2}\left(\mathcal{W}_{0}, \mathbb{F}\right)=\mathbb{F} \bar{\phi}_{0}$, where $\bar{\phi}_{0}$ is the cohomology class of $\phi_{0}$ defined by

$$
\phi_{0}\left(x^{\alpha}\left[\partial_{1}\right]_{\mu}, x^{\beta}\left[\partial_{1}\right]_{\nu}\right)=\delta_{\alpha+\beta, 0}(-1)^{\mu} \mu ! \nu !\left(\begin{array}{c}
\alpha+\mu \\
\mu+\nu+1
\end{array}\right),
$$

for $\alpha, \beta \in \Gamma \subseteq \mathbb{F}, \mu, \nu \in \mathbb{Z}_{+}$, where $\left[\partial_{1}\right]_{\mu}=\partial_{1}\left(\partial_{1}-1\right) \cdots\left(\partial_{1}-\mu+1\right)$.

(2) If $\ell_{4}^{\prime}=\ell_{3}=1$, then for any $\gamma \in \Gamma$, there exists a cohomology class $\bar{\phi}_{\gamma} \in H^{2}\left(\mathcal{W}_{0}, \mathbb{F}\right)$ defined by

$$
\phi_{\gamma}\left(x^{\alpha, i} \partial^{\mu}, x^{\beta, j} \partial^{\nu}\right)=\delta_{\alpha+\beta, \gamma}(-1)^{\mu} \mu ! \nu ! \sum_{s=0}^{\mu+\nu+1}\left(\begin{array}{l}
i \\
s
\end{array}\right) \frac{\alpha^{\mu+\nu+1-s}}{(\mu+\nu+1-s) !} \cdot \frac{\gamma^{s-i-j-1}}{(s-i-j-1) !},
$$

for all $(\alpha, i, \mu),(\beta, j, \nu) \in \Gamma \times \mathbb{Z} \times \mathbb{Z}_{+}$, where as in [S2], $\frac{1}{k !}$ is understood as zero when $k<0$, and when $\alpha$ is taken value 0 , it is understood as $\lim _{\alpha \rightarrow 0} \alpha$ (thus in particular, $\alpha^{\mu+\nu+1-r}=1$ if $\mu+\nu+1-r=0$ and $\alpha=0)$. Furthermore $H^{2}\left(\mathcal{W}_{0}, \mathbb{F}\right)=\prod_{\gamma \in \Gamma} \mathbb{F} \bar{\phi}_{\gamma}$ is a direct product.

(3) If $\ell_{1}+\ell_{2} \geq 1$ or $\ell_{4}^{\prime} \geq 2$, then $H^{2}\left(\mathcal{W}_{0}, \mathbb{F}\right)=0$.

Remark 3.2. (1) It is proved in [S2] that $\phi_{\gamma}$ is in fact a 2-cocycle of the associative algebra $\mathcal{W}_{0}$, satisfying

$$
\phi_{\gamma}(a, b c)+\phi_{\gamma}(b, c a)+\phi_{\gamma}(c, a b)=0 \text { for } a, b, c \in \mathcal{W}_{0} .
$$

(2) In the case of Theorem 3.1(1), we can suppose $1 \in \Gamma$ (see e.g., [SZ2]). Then we can define the derivative $\frac{d}{d x}$ by $\frac{d}{d x} x^{\alpha}=\alpha x^{\alpha-1}$. We have $\left[\partial_{1}\right]_{\mu}=x^{\mu}\left(\frac{d}{d x}\right)^{\mu}$ and (3.3) becomes

$$
\phi_{0}\left(x^{\alpha+\mu}\left(\frac{d}{d x}\right)^{\mu}, x^{\beta+\nu}\left(\frac{d}{d x}\right)^{\nu}\right)=\delta_{\alpha+\beta, 0}(-1)^{\mu} \mu ! \nu !\left(\begin{array}{c}
\alpha+\mu \\
\mu+\nu+1
\end{array}\right) \text { for } \alpha, \beta \in \Gamma, \mu, \nu \in \mathbb{Z}_{+}
$$

(this 2-cocycle for the case $\Gamma=\mathbb{Z}$ (the classical Weyl algebra) seems to appear first in [KP]). We prove as follows that $\phi_{0}$ also satisfies (3.4): First by (3.5), we have

$$
\phi_{0}\left(x^{\alpha+\mu}\left(\frac{d}{d x}\right)^{\mu}, x^{\beta+\nu}\left(\frac{d}{d x}\right)^{\nu}\right)=(-1)^{\nu+1} \frac{\mu ! \nu !}{(\mu+\nu+1) !} \phi_{0}\left(x^{1}, x^{\alpha+\mu}\left(\frac{d}{d x}\right)^{\mu+\nu+1}\left(x^{\beta+\nu}\right)\right) .
$$


Using this and $x^{\alpha+\mu}\left(\frac{d}{d x}\right)^{\mu} x^{\beta+\nu}\left(\frac{d}{d x}\right)^{\nu}=\sum_{\lambda \in \mathbb{Z}_{+}}\left(\begin{array}{c}\mu \\ \lambda\end{array}\right)[\beta+\nu]_{\lambda} x^{\alpha+\beta+\mu+\nu-\lambda}\left(\frac{d}{d x}\right)^{\mu+\nu-\lambda}$ (where $[\beta+\nu]_{\lambda}$ is a similar notation to $\left[\partial_{1}\right]_{\lambda}$, cf. $\left.(3.2),(1.16)\right)$, we have

$\phi_{0}\left(a\left(\frac{d}{d x}\right)^{\mu}, b\left(\frac{d}{d x}\right)^{\nu} c\left(\frac{d}{d x}\right)^{\lambda}\right)=\sum_{s=0}^{\nu}(-1)^{\nu+\lambda+1-s} \frac{\mu !(\nu+\lambda-s) !}{(k+1-s) !}\left({ }_{s}^{\nu}\right) \phi_{0}\left(x^{1}, a\left(\frac{d}{d x}\right)^{k+1-s}\left(b\left(\frac{d}{d x}\right)^{s}(c)\right)\right)$

for $a, b, c \in \mathcal{A}_{\overline{0}}$ (cf. (1.11)), where $k=\mu+\nu+\lambda$. Using shifted version of (3.7), we have

$$
\begin{aligned}
& \phi_{0}\left(b\left(\frac{d}{d x}\right)^{\nu}, c\left(\frac{d}{d x}\right)^{\lambda} a\left(\frac{d}{d x}\right)^{\mu}\right)=\sum_{s=0}^{\lambda}(-1)^{\nu+s} \frac{\nu !(\lambda+\mu-s) !}{(k+1-s) !}(\underset{s}{\lambda}) \phi_{0}\left(x^{1}, a\left(\frac{d}{d x}\right)^{s}\left(\left(\frac{d}{d x}\right)^{k+1-s}(b) c\right)\right), \\
& \phi_{0}\left(c\left(\frac{d}{d x}\right)^{\lambda}, a\left(\frac{d}{d x}\right)^{\mu} b\left(\frac{d}{d x}\right)^{\nu}\right)=\sum_{s=0}^{\mu}(-1)^{\lambda} \frac{\lambda !(\nu+\mu-s) !}{(k+1-s) !}(\underset{s}{\mu}) \phi_{0}\left(x^{1}, a\left(\frac{d}{d x}\right)^{s}(b)\left(\frac{d}{d x}\right)^{k+1-s}(c)\right) .
\end{aligned}
$$

Denote the right-hand sides of (3.7)-(3.9) by $\sum_{s=0}^{k+1} d_{p, s} \phi\left(t^{1}, a\left(\frac{d}{d x}\right)^{s}(b)\left(\frac{d}{d x}\right)^{k+1-s}(c)\right)$ for $p=$ 1,2 and 3 respectively. Using $(1+x)^{k+1-s}(1+x)^{-(\lambda+1)}=(1+x)^{\mu+\nu-s}$, we deduce the binomial formula $\sum_{q}(-1)^{q}\left(\begin{array}{c}k+1-s \\ \nu-q\end{array}\right)\left(\begin{array}{c}\lambda+q \\ q\end{array}\right)=\left(\begin{array}{c}\mu+\nu-s \\ \nu\end{array}\right)$. From this, we can deduce that if $s \leq \mu$, then

$$
d_{1, s}=\sum_{q=0}^{\nu}(-1)^{\nu+\lambda+1-q} \frac{\mu !(\nu+\lambda-q) !}{(k+1-q) !}\left(\stackrel{\nu}{q}^{\nu}\left(\begin{array}{c}
k+1-q \\
s
\end{array}\right)=(-1)^{\lambda+1} \frac{\lambda !(\nu+\mu-s) !}{(k+1-s) !}\left(\begin{array}{c}
\mu \\
s
\end{array}\right)=-d_{3, s}\right.
$$

and $d_{2, s}=0$; and if $\mu<s \leq \mu+\nu$, then $d_{1, s}=d_{2, s}=d_{3, s}=0$; and if $\mu+\nu<s \leq k+1$, then

$$
\begin{aligned}
d_{1, s} & =\sum_{q=0}^{\nu}(-1)^{\nu+\lambda+1-q} \frac{\mu !(\nu+\lambda-q) !}{(k+1-q) !}(\underset{q}{\nu})\left(\begin{array}{l}
k+1-q \\
s
\end{array}\right) \\
& =\sum_{q=0}^{\lambda}(-1)^{\nu+q+1} \frac{\nu !(\lambda+\mu-q) !}{(k+1-q) !}\left(\begin{array}{l}
\lambda \\
q
\end{array}\right)\left(\begin{array}{c}
q+1-s \\
k+1
\end{array}\right)=-d_{2, s},
\end{aligned}
$$

and $d_{3, s}=0$. This proves that the sum of $(3.7)-(3.9)$ is zero.

Assume $\ell_{5}>0$. Observe that as an associative superalgebra under the product (1.16), $\mathcal{W}$ can be decomposed into the following tensor product of super-subalgebras:

$$
\mathcal{W}=\mathcal{W}_{0} \otimes \mathcal{W}_{1}, \quad \mathcal{W}_{1}=\mathcal{W}_{1,1} \otimes \mathcal{W}_{1,2} \otimes \cdots \otimes \mathcal{W}_{1, \ell_{5}}
$$

where

$$
\mathcal{W}_{1, p}=\operatorname{span}\left\{s_{p}^{m} \check{\partial ̆}_{p}^{n} \mid m, n \in\{0,1\}\right\} \quad \text { (a superalgebra of dimension 4) for } p \in \overline{1, \ell_{5}}
$$

(cf. notations (1.9) and (1.13)). Denote

$$
\mathcal{W}_{p}^{\wedge}=\mathcal{W}_{0} \otimes \mathcal{W}_{1,1} \otimes \cdots \otimes \mathcal{W}_{1, p-1} \otimes \mathcal{W}_{1, p+1} \otimes \cdots \otimes \mathcal{W}_{1, \ell_{5}} \text { for } p \in \overline{1, \ell_{5}}
$$


In the following, an element $v \in \mathcal{W}$ will always mean a homogeneous element with $g(v) \in \mathbb{Z}_{2}$. Observe from (1.16) that

$$
[u, v w]=[u, v] w+(-1)^{g(u) g(v)} v[u, w] \text { for } u, v, w \in \mathcal{W} .
$$

Thus for $a, b \in \mathcal{W}_{0}, u, v \in \mathcal{W}_{1}$ and $y, z \in \mathcal{W}_{p}^{\wedge}$, we have

$$
\begin{aligned}
& {[a u, b v]=[a, b] u v+b a[u, v],} \\
& {\left[y s_{p}, z s_{p}\right]=\left[y \check{\partial}_{p}, z \check{\partial}_{p}\right]=0,} \\
& {\left[y s_{p} \check{\partial}_{p}, z s_{p} \check{\partial}_{p}\right]=\left[y s_{p} \check{\partial}_{p}, z\right]=[y, z] s_{p} \check{\partial}_{p},} \\
& {\left[y s_{p}, z \check{\partial}_{p}\right]=(-1)^{g(z)}\left([y, z] s_{p} \check{\partial}_{p}+(-1)^{g(y) g(z)} z y\right) .}
\end{aligned}
$$

Now suppose $\varphi$ is a 2-cocycle satisfying (2.5)-(2.7).

Lemma 3.3. We have

$$
\begin{gathered}
\begin{aligned}
\varphi(y, z) & =\varphi\left(y s_{p}, z\right)=\varphi\left(y s_{p}, z s_{p}\right)=\varphi\left(y \check{\partial}_{p}, z\right) \\
= & \varphi\left(y \check{\partial}_{p}, z \check{\partial}_{p}\right)=\varphi\left(y s_{p} \check{\partial}_{p}, z s_{p}\right)=\varphi\left(y s_{p} \check{\partial}_{p}, z \check{\partial}_{p}\right)=0,
\end{aligned} \\
(-1)^{g(z)} \varphi\left(y s_{p}, z \check{\partial}_{p}\right)=\varphi\left(y, z s_{p} \check{\partial}_{p}\right)=\varphi\left(y s_{p} \check{\partial}_{p}, z s_{p} \check{\partial}_{p}\right),
\end{gathered}
$$

for $p \in \overline{1, \ell_{5}}$ and $y, z \in \mathcal{W}_{p}^{\wedge}$.

Proof. We have $\varphi(y, z)=\varphi\left(\left[\check{\partial}_{p}, s_{p} y\right], z\right)=(-1)^{1+g(y)} \varphi\left(s_{p} y,\left[\check{\partial}_{p}, z\right]\right)=0$. The other equalities of (3.18) follow from the fact

$$
0=\varphi\left(s_{p} \check{\partial}_{p},\left[y s_{p}^{k} \check{\partial}_{p}^{\mu}, z s_{p}^{k^{\prime}} \check{\partial}_{p}^{\nu}\right]\right)=\left(k+k^{\prime}-\mu-\nu\right) \varphi\left(y s_{p}^{k} \check{\partial}_{p}^{\mu}, z s_{p}^{k^{\prime}} \check{\partial}_{p}^{\nu}\right) \quad(\text { by }(2.4) \text { and }(2.7))
$$

for $k, k^{\prime}, \mu, \nu \in\{0,1\}$. By (2.4) and (2.6), we have

$$
0=\varphi\left(\check{\partial}_{p},\left[y s_{p}, z s_{p} \check{\partial}_{p}\right]\right)=(-1)^{g(y)}\left(\varphi\left(y, z s_{p} \check{\partial}_{p}\right)-(-1)^{g(z)} \varphi\left(y s_{p}, z \check{\partial}_{p}\right)\right)
$$

which gives the first equality of (3.19). Since $\mathcal{W}_{p}^{\wedge}=\left[\mathcal{W}_{p}^{\wedge}, \mathcal{W}_{p}^{\wedge}\right]$, by linearity, we can suppose $z=\left[z_{1}, z_{2}\right]$ for some $z_{1}, z_{2} \in \mathcal{W}_{p}^{\wedge}$ without loss of generality. Then by (3.16) and (2.4),

$$
\begin{aligned}
\varphi\left(y, z s_{p} \check{\partial}_{p}\right) & =\varphi\left(\left[y, z_{1} s_{p} \check{\partial}_{p}\right], z_{2} s_{p} \check{\partial}_{p}\right)+(-1)^{g(y) g\left(z_{1}\right)} \varphi\left(z_{1} s_{p} \check{\partial}_{p},\left[y, z_{2} s_{p} \check{\partial}_{p}\right]\right) \\
& =\varphi\left(\left[y s_{p} \check{\partial}_{p}, z_{1} s_{p} \check{\partial}_{p}\right], z_{2} s_{p} \check{\partial}_{p}\right)+(-1)^{g(y) g\left(z_{1}\right)} \varphi\left(z_{1} s_{p} \check{\partial}_{p},\left[y s_{p} \check{\partial}_{p}, z_{2} s_{p} \check{\partial}_{p}\right]\right) \\
& =\varphi\left(y s_{p} \check{\partial}_{p},\left[z_{1} s_{p} \check{\partial}_{p},, z_{2} s_{p} \check{\partial}_{p}\right]\right)=\varphi\left(y s_{p} \check{\partial}_{p}, z s_{p} \check{\partial}_{p}\right),
\end{aligned}
$$


which gives the second equality of (3.19).

Note that we have

$$
\mathcal{W}_{1}=\left[\mathcal{W}_{1}, \mathcal{W}_{1}\right] \oplus \mathbb{F} u_{1} \text {, where } u_{1}=s_{1} \cdots s_{\ell_{5}} \check{\partial}_{1} \cdots \check{\partial}_{\ell_{5}} \in \mathcal{W}_{1}
$$

We define a linear function $P: \mathcal{W}_{1} \rightarrow \mathbb{F}$ by setting

$$
P\left(u_{1}\right)=1 \text { and } P\left(\left[\mathcal{W}_{1}, \mathcal{W}_{1}\right]\right)=0 \text {. }
$$

Then by the second equation of (3.21), we have

$$
P(u v)=(-1)^{g(u) g(v)} P(v u) \text { for } \quad u, v \in \mathcal{W}_{1} .
$$

Define a bilinear function $\phi: \mathcal{W}_{0} \times \mathcal{W}_{0} \rightarrow \mathbb{F}$ by setting

$$
\phi(a, b)=\varphi\left(a u_{1}, b u_{1}\right)=\varphi\left(a, b u_{1}\right) \quad \text { for } \quad a, b \in \mathcal{W}_{0}
$$

where the second equality follows from Lemma 3.3 and the fact that $u_{1} u_{1}=u_{1}$. We have

Lemma 3.4. $\phi$ is a 2-cocycle on $\mathcal{W}_{0}$ satisfying (2.5) and (2.6) (with $\ell$ replaced by $\ell_{4}^{\prime}$ ). In particular $\phi=c \phi_{0}$ for some $c \in \mathbb{F}$ if $\ell_{4}^{\prime}=\ell_{3}=1$, or $\phi=\sum_{\gamma \in \Gamma} c_{\gamma} \phi_{\gamma}$ for some $c_{\gamma} \in \mathbb{F}$ if $\ell_{4}^{\prime}=\ell_{3}=1$, or $\phi=0$ if $\ell_{2}^{\prime} \geq 1$ or $\ell_{4}^{\prime} \geq 2$.

Proof. The first statement can be verified directly, the second follows from [S2] (we remark that although $\sum_{\gamma \in \Gamma} c_{\gamma} \phi_{\gamma}$ may be an infinite sum, it is summable in the sense that when it applies to any $(a, b)$ for $a, b \in \mathcal{W}_{0}$, there are only finite many nonzero terms).

Our main result of this paper is the following.

Theorem 3.5. (1) Suppose $\ell_{4}^{\prime}=\ell_{4}=1$. Then $H^{2}(\mathcal{W}, \mathbb{F})=\mathbb{F} \bar{\varphi}_{0}$, where $\varphi_{0}$ is defined by

$$
\varphi_{0}(a u, b v)=\phi_{0}(a, b) P(u v) \quad \text { for } \quad a, b \in \mathcal{W}_{0}, u, v \in \mathcal{W}_{1},
$$

and $\phi_{0}$ is defined by (3.2).

(2) Suppose $\ell_{4}^{\prime}=\ell_{3}=1$. Then for any $\gamma \in \Gamma$, there corresponds a cohomology class $\bar{\varphi}_{\gamma} \in H^{2}(\mathcal{W}, \mathbb{F})$ defined by

$$
\varphi_{\gamma}(a u, b v)=\phi_{\gamma}(a, b) P(u v) \quad \text { for } \quad a, b \in \mathcal{W}_{0}, u, v \in \mathcal{W}_{1}
$$

and $\phi_{\gamma}$ is defined by (3.3). Furthermore, $H^{2}(\mathcal{W}, \mathbb{F})$ is a direct product:

$$
H^{2}(\mathcal{W}, \mathbb{F})=\prod_{\gamma \in \Gamma} \mathbb{F} \bar{\varphi}_{\gamma}
$$


(3) If $\ell_{2}^{\prime} \geq 1$ or $\ell_{4}^{\prime} \geq 2$, then $H^{2}(\mathcal{W}, \mathbb{F})=0$.

Proof. The result follows from Theorem 3.1 if $\ell_{5}=0$. Thus assume $\ell_{5} \geq 1$. First we verify that in case of $\ell_{4}^{\prime}=\ell_{4}=1$, a bilinear function $\varphi_{0}$ defined by (3.24) is a nontrivial 2cocycle on $\mathcal{W}$ : The super-skew-symmetry follows from the skew-symmetry of $\phi_{0}$ and (3.22). By (3.14), we have

$$
\begin{aligned}
\varphi_{0}(a u,[b v, c w]) & =\varphi_{0}(a u,[b, c] v w+c b[v, w]) \\
& =\phi_{0}(a,[b, c]) P(u v w)+\phi_{0}(a, c b) P(u[v, w]),
\end{aligned}
$$

for $a, b, c \in \mathcal{W}_{0}, u, v, w \in \mathcal{W}_{1}$. Now the super-Jacobi identity follows from (3.4), (3.22) and (3.27) (together with its shifted version). Clearly, $\varphi_{0}$ is nontrivial since $\phi_{0}$ is nontrivial. Similarly, in case of $\ell_{4}^{\prime}=\ell_{3}=1$, since (3.27) still holds with $\varphi_{0}$ replaced by $\varphi_{\gamma}$ for any $\gamma \in \Gamma$, one can prove that $\varphi=\sum_{\gamma \in \Gamma} c_{\gamma} \varphi_{\gamma}$ is a nontrivial 2-cocycle on $\mathcal{W}$, where $\varphi_{\gamma}$ is defined in (3.25), and $c_{\gamma} \in \mathbb{F}$ for $\gamma \in \Gamma$ such that $c_{\gamma} \neq 0$ for at least one $\gamma \in \Gamma$. In particular, the right-hand side of (3.26) is a direct product.

Now suppose $\varphi$ is a 2-cocycle satisfying (2.5)-(2.7). For any

$$
u=s_{1}^{j_{1}} \cdots s_{\ell_{5}}^{j_{\ell_{5}}} \check{\partial}_{1}^{\mu_{1}} \cdots \check{\partial}_{\ell_{5}}^{\mu_{\ell_{5}}} \in \mathcal{W}_{1}, \quad \text { where } j_{p}, \mu_{p} \in\{0,1\},
$$

we define its support to be $\operatorname{supp}(u)=\left\{p \in \overline{1, \ell_{5}} \mid\left(j_{p}, \mu_{p}\right) \neq 0\right\}$. We want to prove

$$
\varphi(a, b u)=0 \text { for } a, b \in \mathcal{W}_{0}, u \in\left[\mathcal{W}_{1}, \mathcal{W}_{1}\right]
$$

Suppose $u \in\left[\mathcal{W}_{1}, \mathcal{W}_{1}\right]$ is as in (3.28), then $u \neq u_{1}$ by (3.20). Thus there exists $p \in \overline{1, \ell_{5}}$ such that $u=u^{\prime} u^{\prime \prime}$ for some $u^{\prime} \in \mathcal{W}_{p}^{\wedge}$ and $u^{\prime \prime}=1, s_{p}$ or $\check{\partial}_{p}$. Now take $y=a, z=b u^{\prime} \in \mathcal{W}_{p}^{\wedge}$, by (3.18), we obtain (3.29). Let $\phi$ be defined as in (3.23). By using (3.19), (3.21), (3.23), (3.29) and induction on the support size \# $\operatorname{supp}(u)$, one can similarly prove

$$
\varphi(a u, b v)=\varphi(a, b u v)=\phi(a, b) P(u v) \text { for } a, b \in \mathcal{W}_{0}, u, v \in \mathcal{W}_{1}
$$

For example, if $\# \operatorname{supp}(u)=0$ then (3.30) follows from (3.21), (3.23) and (3.29).

Now the theorem follows from (3.30) and Lemma 3.4.

\section{References}

[BKV] B. Bakalov, V.G. Kac, A.A. Voronov, "Cohomology of conformal algebras," Comm. Math. Phys. 200 (1999), 561-598. 
[K1] V.G. Kac, Infinite Dimensional Lie Algebras, 3rd ed., Combridge Univ. Press, 1990.

[K2] V.G. Kac, "Lie superalgebras," Adv. Math. 26 (1977), 8-96.

[KP] V.G. Kac, D.H. Peterson, "Spin and pepresentation of infinite dimensional Lie algebras and groups," Proc. Nat. Acad. Sci. U.S.A. 78 (1981), 3308-3312.

[L] W. Li, "2-Cocycles on the algebra of differential operators," J. Algebra 122 (1989), 64-80.

[LW] W. Li, R.L. Wilson, "Central extensions of some Lie algebras," Proc. Amer. Math. Soc. 126 (1998), 2569-2577.

[ScZ1] M. Scheunert, R.B. Zhang, "Cohomology of Lie superalgebras and their generalizations," J. Math. Phys. 39 (1998), 5024-5061.

[ScZ2] M. Scheunert, R.B. Zhang, "The second cohomology of $s l(m / 1)$ with coefficients in its enveloping algebra is trivial," Lett. Math. Phys. 47 (1999), 33-48.

[S1] Y. Su, "2-Cocycles on the Lie algebras of all differential operators of several indeterminates," (Chinese) Northeastern Math. J. 6 (1990), 365-368.

[S2] Y. Su, "2-cocycles on the Lie algebras of generalized differential operators", Comm. Algebra 30 (2002), 763-782.

[S3] Y. Su, "Classification of quasifinite modules over the Lie algebras of Weyl type," Adv. Math. 174 (2003), 57-68.

[S4] Y. Su, "Low dimensional cohomology of general conformal algebras gc , , J. Math. Phys. 45 (2004), 509-524.

[SXZ] Y. Su, X. Xu and H. Zhang, "Derivation-simple algebras and the structures of Lie algebras of Witt type," J. Algebra 2000 (233), 642-662.

[SZ1] Y. Su, K. Zhao, "Simple Lie algebras of Weyl type," Science in China A 44 (2001), 419-426.

[SZ2] Y. Su, K. Zhao, "Isomorphism classes and automorphism groups of algebras of Weyl type," Science in China A 45 (2002), 953-963.

[SZ3] Y. Su, K. Zhao, "Second cohomology group of generalized Witt type Lie algebras and certain reperesentations," Comm. Alegrba 30 (2002), 3285-3309.

[SZZ] Y. Su, K. Zhao and L. Zhu, "Simple Lie color algebras of Weyl types," Israel J. Math. in press.

[X1] X. Xu, "New generalized simple Lie algebras of Cartan type over a field with characteristic 0," J. Algebra 224 (2000), 23-58.

[X2] X. Xu, "Generalizations of Block algebras," Manuscripta Math. 100 (1999), 489-518.

[X3] X. Xu, "Simple conformal superalgebras of finite growth," Algebra Colloquium 7 (2000), 205-240.

[X4] X. Xu, "Equivalence of conformal superalgebras to Hamiltonian superoperators," Algebra Colloquium 8 (2001), 63-92.

[X5] X. Xu, "Quadratic Conformal Superalgebras," J. Algebra 231 (2000), 1-38.

[Z] K. Zhao, "Simple algebras of Weyl type II," Proc. Amer. Math. Soc. 130 (2002), 13231332. 